\title{
CALIDAD DE CUIDADOS DE ENFERMERÍA A LOS PACIENTES TRAUMATIZADOS POR ACCIDENTES DE TRÁNSITO EN CATAMARCA, ARGENTINA
}

Graciela García ${ }^{1}$ Ruth Fernández ${ }^{2}$ Gabriel Acevedo ${ }^{3}$ Rolando Montenegro ${ }^{4}$

${ }^{1}$ Licenciada en Enfermería. Facultad de Ciencias de la Salud Universidad Nacional de Catamarca, Argentina.

Dirección de Enfermería, Ministerio de Salud de Catamarca, Argentina. ${ }^{2}$ Doctora de Medicina, Profesora de la Escuela de Salud Pública de la Facultad e Ciencias Médicas, Universidad Nacional de Córdoba, Argentina. ${ }^{3}$ Doctor en Medicina,

Profesor de la Escuela Salud Pública de la Facultad de Ciencias Médicas,

Universidad Nacional de Córdoba, Argentina. ${ }^{4}$ Doctor en Medicina, Especialista en Traumatología, Hospital de Urgencias de la Ciudad de Córdoba, Argentina. Correspondencia: gracielagarcia_04@hotmail.

Trabajo recibido: 30 de Julio de 2017. Aprobado: 30 de Octubre de 2017.

\author{
QUALITY OF NURSING CARE TO PATIENTS \\ TRAUMATIZED IN TRAFFIC ACCIDENTS IN \\ CATAMARCA, ARGENTINA
}

\section{QUALIDADE DE CUIDADO DE ENFERMA- GEM PARA PACIENTES TRAUMATIZADOS POR ACIDENTES DE TRÂNSITO EM CATA- MARCA, ARGENTINA}

\section{Resumen}

Objetivo: determinar la calidad de atención de enfermería y satisfacción de pacientes traumatizados, Servicio de Traumatología del Hospital Interzonal San Juan Bautista. Método: observacional, descriptivo corte transversal. Se aplicó encuesta estructurada para caracterización socio demográfica y nivel de formación del personal, guía de observación de procedimientos que realizó enfermería a pacientes traumatizados, aplicando coeficiente positivo a procedimientos realizados bien.

Se observó al 100\% (46), de enfermeras del Servicio y $100 \%$ (460) de personas traumatizadas durante 2016 Resultados: El análisis socio demográfico del personal determinó que $72 \%$ son femeninos. La media de edad del personal, fue de 40,28 $\pm 0,29$ años, para ambos sexos El nivel de formación: 24 auxiliar de enfermería, 15 enfermeros, 7 licenciados en enfermería.

Los procedimientos independientes: higiene, eliminaciones de aparatos urinario y digestivo registraron 
puntaje negativo, los tratamientos interdependientes obtuvieron puntuaciones positivas. La mayoría de los pacientes valoraron bien los cuidados que recibieron,cortesía, rapidez con que satisficieron sus necesidades.

Palabras clave: Enfermera, Accidentes de tránsitos, Hospitalización.

\section{Abstract}

Objective: To determine quality of nursing care and satisfaction of traumatized patients at the Orthopedics Service, Hospital Interzonal San Juan Bautista. Method: Observational, descriptive, cross sectional study. Structured interview was applied for demographic characterization and staff educational level, guide of observation of procedures carried out in nursing to traumatized patients, applying positive coefficients to well done procedures. It was observed to $100 \%, 46$ nurses from the Service and 460 traumatizedpeople during 2016.

Results: Socio demographic analysis of staff determined that $72 \%$ are women. Staff average age was $40.28 \pm 0.29$ for both sexes.Educational level: 24 auxiliary nurses , 15 nurses, registered nurses.Independent procedures: hygiene, urinary and digestive system evacuation got negative scores; interdependent procedures got positive scores. Most patients graded well the care received, courtesy and speed to satisfy needs.

Key words: Nurse, Traffic accidents, Hospitalization.

\section{Resumo}

Objetivo: determinar a qualidade do atendimento de enfermagem e satisfação de pacientes traumatizados, Serviço de Traumatologia do Hospital Interzonal de San Juan Bautista.

Método: observacional, descritivo corte transversal. Foi aplicada uma enquete estruturada para caracterização sociodemográfica e nível de treinamento de pessoal, guia de desempenho de procedimentos que realizou Enfermagem para pacientes traumatizados, aplicando coeficiente positivo para aqueles corretamente realizados.Observou-se em 100\% (46) de enfermeiras do Serviço e 100\% (460) de pessoas traumatizadas durante 2016 |Resultados: A análise sociodemográfica da equipe determinou que $72 \%$ são mulheres. A idade média da equipe foi de 40,28 $\pm 0,29$ anos, para ambos os sexos O nível de treinamento: 24 auxiliares de enfermagem, 15 enfermeiros, 7 bacharéis em enfermagem.

Os procedimentos independentes: higiene, eliminação de detritos urinários e digestivos registraram pontuação negativa, os tratamentos interdependentes obtiveram pontuação positiva A maioria dos pacientes avaliou positivamente o cuidado que receberam, cortesia, velocidade com a qual as suas necessidades.foram satisfeitas.

Palavras chave: Enfermeiras, Acidentes de trânsito, Hospitalização.

\section{Introducción}

Los accidentes de tránsito generan entidades nosológicas incluidas en la Clasificación Internacional de Enfermedades (CIE 10), propuesta por la Organización Mundial de la Salud, ubicadas entre los códigos V01 - V89, que asumidas como enfermedad traumática o trauma, han permitido homogeneizar la denominación permitiendo comparar los datos de países desarrollados o en vía de desarrollo $(1,2)$. A su vez, el trauma, como entidad nosológica presenta tres fases, el pre trauma, el trauma y el pos - trauma. Esta última fase se corresponde a la atención intrahospitalaria, donde se determina las mejores formas de tratamientos para recuperar la fisiología, que pone en riesgo la vida del paciente, y/o recuperar al máximo las funciones perdidas. Es decir que, en esta etapa, la atención apropiada favorece la reducción de las secuelas y discapacidades, minimiza el sufrimiento 
y facilita la adaptación de los pacientes a su entorno, aplicándose así el concepto de prevención terciaria (3).

El trauma y sus consecuencias tales como la muerte, las discapacidades psicofísicas temporales o permanentes y el elevado costo socioeconómico, afectan tanto la vida de las personas, como al sistema organizativo de una ciudad, e influye negativamente en su calidad de vida y la de sus habitantes (4).

El desarrollo de los sistemas o servicios de trauma en nuestro país llevó a una reducción significativa, en el número de las muertes prevenibles postraumáticas. En la actualidad se considera aceptable, una tasa de muerte prevenible menor que el $2 \%$ como el ideal de un sistema de trauma. Sin embargo, aún fallecen pacientes que desarrollan complicaciones que, podrían evitarse o prevenirse. Estos errores se producen en fases diferentes de la atención del paciente traumatizado, tales como la reanimación, la fase operatoria y la de cuidados críticos $(5,6)$. Por otra parte en el Servicio de Trauma de los Hospitales, las causas de muertes prevenibles incluyen errores en el juicio clínico - quirúrgico o en las técnicas instituidas. Esta concepción es útil para monitorear el desempeño de los integrantes del equipo de salud, a través de la evolución del paciente, lo cual está asociado al control de calidad de la atención $(7,8)$

La enfermera Virginia Henderson define en su teoría la calidad de atención de enfermería como la consecución de conjuntos de características y acciones que posibilitan la restauración de la salud en cada paciente $(9,10)$.

Desde la perspectiva de la atención hospitalaria la conceptualización de atención de calidad tiene que ver con una dimensión técnica, que consiste en la mejor aplicación del conocimiento y la tecnología disponible a favor de los pacientes (gestión de cuidados, prestaciones y desempeño). En sí, una dimensión social que mide la satisfacción del paciente y las relaciones humanas y, además, una dimensión de calidad que responde al grado en que el servicio satisface las necesidades del destinatario (11). Esta concepción, que analiza la atención que se brinda en los servicios sanitarios se relaciona al concepto de Donabedian Vuori y colaboradores para abordar la calidad en las acciones de asistencia (12). La satisfacción y la percepción de la calidad en las instituciones de salud, por parte del cliente es bastante más compleja que en otras instituciones de servicio: un óptimo resultado asistencial no siempre es sinónimo de satisfacción, a pesar que calidad no significa necesariamente mejor, sino el mejor nivel de respuesta a las expectativas del usuario, con el menor riesgo posible (13).

En Catamarca (Argentina), los accidentes son factores de morbi - mortalidad prematura, discapacidad física, enfermedad y un elevado costo asistencial. Los pacientes ingresan por el servicio de emergencia donde permanecen internados en un promedio de 24 a 48 horas, y luego de acuerdo a su evolución son derivados al Servicio de Traumatología, para la continuidad de su tratamiento. Los pacientes durante este período, aún sin estar calificados para evaluar los aspectos intrínsecos de los tratamientos brindados por los integrantes del equipo de salud son conscientes de sus derechos y plantean con mayor exigencia las bondades perceptibles de la atención sanitaria. La atención de enfermería cobra un interés especial en los pacientes con trauma. La Organización Mundial de la Salud estimula esfuerzos colaborativos para avanzar en la investigación de Atención Esencial del Trauma combinando las perspectivas de la salud pública y el área clínica de los sistemas de trauma (14). En los recursos descriptos como esenciales se encuentran los referidos al área de atención de enfermería, con el consecuente registro del rendimiento en esa área. Asimismo la Organización Mundial de la Salud define calidad de atención en enfermería como el alto nivel de excelencia profesional, uso eficiente de recursos, mínimo de riesgos de complicaciones, con un alto grado de satisfacción por parte del paciente. Así, la calidad tiene dos aspectos básicos: 1) la calidad técnica o intrínseca referida a las 
características técnicas de la atención y 2) la calidad percibida en relación a la impresión que los usuarios tienen sobre la idoneidad de un servicio para satisfacer sus expectativas (15). Dado que el tratamiento avanzado de las heridas se sustenta en el conocimiento del proceso de cicatrización (16) y en los factores que le son adversos; el personal debe realizar los procedimientos con técnicas y ordenamiento, para la optimización de la calidad de atención disminuyendo así los tiempos de tratamiento y los costos (17).

En función de lo expuesto, el objetivo del presente trabajo fue determinar la calidad de atención de enfermería y grado de satisfacción de pacientes traumatizados, en el Servicio de Traumatología del Hospital Interzonal San Juan Bautista Catamarca, Argentina.

\section{Material y Métodos}

El diseño de la investigación fue observacional, descriptivo y de corte transversal.

Unidades de observación en el personal de enfermería: se estudió al 100\% del personal de enfermería que se desempeñaba en el Servicio de Traumatología del Hospital Interzonal San Juan Bautista (46 empleados) durante el período correspondiente al año 2012. Se efectuaron diez observaciones por cada técnica de ejecución prevista en la Guía de Observación.

Unidades de encuestas de pacientes: fue el $100 \%$ de las personas traumatizadas, por accidentes de tránsitos derivadas al Servicio de Traumatología, que permanecieron internadas y que ingresaron conscientes, durante el mismo período. Total de pacientes incluidos en el estudio fue de 460.

Los datos que sustentan este estudio se recopilaron a través de tres instrumentos:

a) Una encuesta estructurada para identificar las características sociodemográficas del personal de enfermería, la cual incluyó sexo, edad, antigüedad, nivel de escolaridad, formación específica, y capacitación.

b) Una guía de observación de las acciones que dedica el personal de enfermería a los pacientes con lesiones externas, por accidentes de tránsitos. Se registró además el turno de trabajo en el que se efectuaba la observación. La guía fue adaptada del Libro Calidad Total en Atención Primaria de Salud (18). En la misma se asignaron coeficientes positivos cuando los procedimientos fueron realizados en forma correcta, y negativos cuando el procedimiento fue realizado incorrectamente. Las variables involucradas en la misma se relacionaron con los aspectos técnicos tales como valoración de signos vitales, administración de medicamentos, aplicación de precauciones universales de bioseguridad, higiene y confort, colocación de venoclisis, acciones de enfermería en las eliminaciones de aparato urinario y digestivo, teniendo en cuenta la preparación de los respectivos equipos, la explicación del procedimiento al paciente, la ejecución de los procedimientos y su registro, la comodidad percibida por el paciente y el acondicionamiento del equipo. Las observaciones se registraron como $\mathrm{Si}$ o No de acuerdo a que se cumplieran o no correctamente los procedimientos.

Este diseño contempló además el cálculo de un coeficiente el cual $\mathrm{P}=3(1)+3(2)+2(3)+$ $3(4)+2(5)+2(6)+2(7)+3(8)+3(9)+3(10)+2(11)+1(12)+1(13)+2(14)+2(15)$ $+2(16)+2(17)+2(18)=40$ puntos

Cuando los aspectos técnicos observados se cumplieron correctamente en su totalidad se obtuvo un valor de Coeficiente positivo, en el caso que se produjese la omisión en uno de los pasos observados, el mismo dio como resultado un coeficiente negativo.

c) Una encuesta para valorar el nivel de satisfacción de los pacientes. Para la construcción de la encuesta se empleó la propuesta de Richard Gerson (19), la cual presenta un conjunto 
de ítems que miden la reacción del sujeto en cinco categorías: Excelente, Muy Bueno, Bueno, Aceptable, Deficiente.

Así, los aspectos evaluados en la encuesta de satisfacción de los pacientes comprendieron tanto las de relaciones con el profesional como de las acciones técnicas profesionales percibidas por el paciente.

Análisis estadístico de los datos: El análisis de los datos fue efectuado de acuerdo a la naturaleza de las variables. Para la descripción de las variables mensurables se presentaron sus medidas de resumen y las variables categóricas fueron expresadas a partir de las frecuencias de las mismas. Las comparaciones de frecuencias fueron efectuadas por datos categorizados y las asociaciones entre las variables mediante análisis multivariado, se consideraron en todos de los casos con un nivel de significación de 0,05.

Consideraciones éticas: En la presente investigación se tuvieron en cuenta los principios éticos para trabajar con seres humanos, implementándose el consentimiento informado tanto en los profesionales como en los pacientes involucrados en el estudio, según las recomendaciones de las normas nacionales e internacionales.

\section{Resultados}

El análisis de las características socio demográficas del personal de enfermería del Servicio de Traumatología del Hospital Interzonal San Juan Bautista reveló que de los 46 integrantes del personal de enfermería que se desempeñan en el Servicio de Traumatología, la mayor proporción corresponde al sexo femenino con el $72 \%$ de la muestra $(p<0,0001)$. En el caso del personal de enfermería del sexo masculino el grupo más representativo tiene entre 38 a 42 años.

El valor de la media de edad del grupo analizado fue de 40,28 \pm 0,29 años, siendo similar para varones y mujeres.

Al evaluar el nivel de instrucción del personal del Servicio de Traumatología del Hospital se observó que la mayoría de ellos cumplieron los estudios secundarios $(93,48 \%)$, persistiendo aún una frecuencia de 3 integrantes que poseen sólo el nivel primario $(6,52 \%)$. En la formación específica se detectó que el Enfermero/a, presenta mayor frecuencia que los Auxiliares de Enfermería y los Licenciados en Enfermería (50\%, 32,61\% y 17,39\% respectivamente) $(\mathrm{p}<0,02)$.

Para evaluar los procedimientos técnicos propios de la enfermería se realizaron 10 observaciones, a cada personal de enfermería del nivel operativo, en los diferentes turnos planificados, aplicando la guía de observación. El primer aspecto analizado fue la valoración de los signos vitales, en donde se evaluó si el personal de enfermería realizaba los controles en forma completa. El análisis mostró que 26 enfermeros controlaron en forma completa y aplicaron los procedimientos en forma correcta, siendo 20 los agentes que no cumplieron con los procedimientos técnicos adecuados. Respecto a la verificación de la ingesta del medicamento, 18 enfermeros la efectuaron, mientras que los 28 restantes no comprobaron que el paciente deglutiera la medicación.

Tal como se observa en la figura 1, el procedimiento de control de venoclisis fue el de mayor frecuencia positiva, continuando en orden de frecuencia, la adecuada colocación de marbetes en los frascos de soluciones de los planes de hidratación, y finalmente la adecuada administración parenteral de los medicamentos y aplicación de las normas de bioseguridad.

Las observaciones realizadas para determinar la calidad de los procedimientos de enfermería fueron evaluadas en relación al nivel de formación obtenido por el personal. 


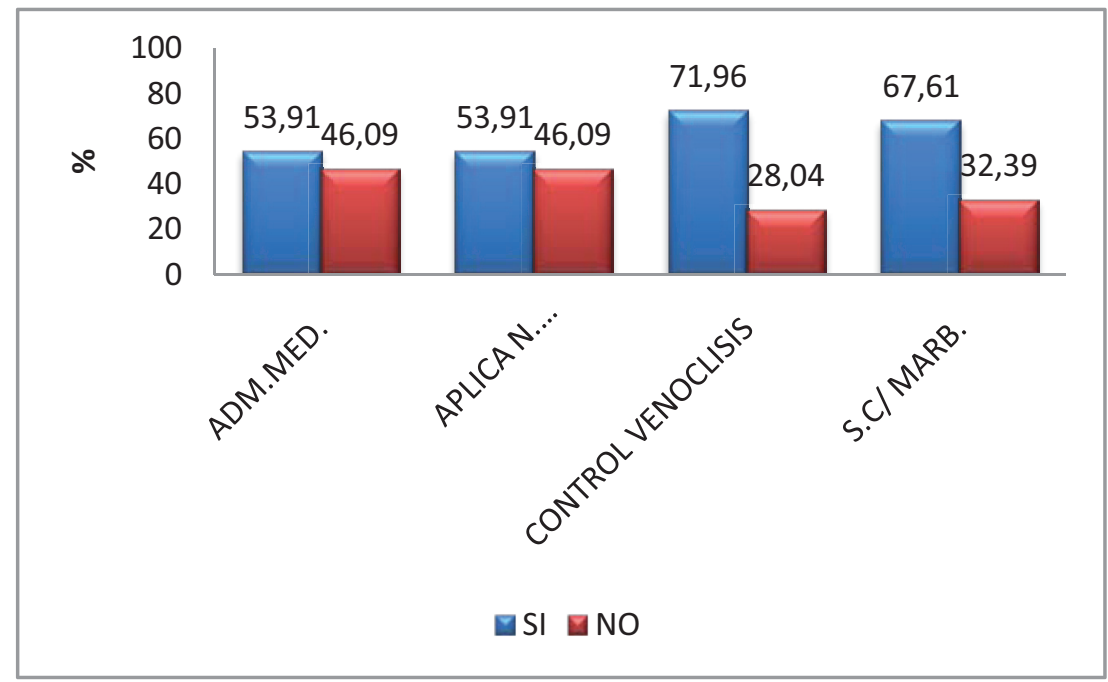

Figura $\mathrm{N}^{\mathrm{o}}$ 1: Frecuencias observadas en administración de medicamentos, aplicación de normas de bioseguridad, control de venoclisis, soluciones con marbetes, del personal de enfermería del Servicio de Traumatología del Hospital Interzonal San Juan Bautista.

Referencia: ADM.MED.: administración de medicamentos. APLICA N. BIOSEGURIDAD: aplica normas de bioseguridad, S. C/ MARB.: soluciones con marbetes.

Así, como resultado de analizar la valoración de los signos vitales según el nivel de formación, reveló que el personal que cuenta con nivel profesional (Enfermera/o y Licenciada/o en Enfermería) controla con mayor frecuencia los signos vitales que los auxiliares de enfermería $(\mathrm{p}<0,0001)$.

En lo que refiere a la instalación de las soluciones de los planes de hidrataciones, el análisis reveló que el licenciado en enfermería es el personal que siempre efectúa el control adecuado, $(\mathrm{p}<0,0001)$.

Cuando se preguntó cómo fue el proceso de admisión que les brindó el personal de enfermería, 271 pacientes expresaron que fue bueno, mientras 105 consideraron que fue aceptable (Figura 2).

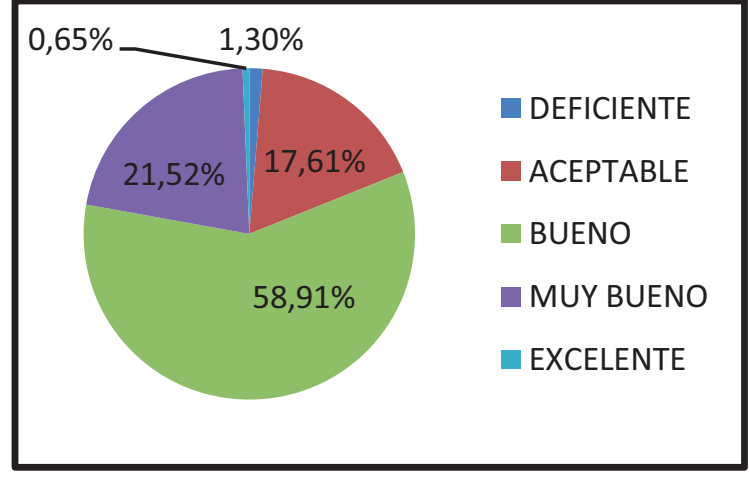

Figura $\mathrm{N}^{\mathrm{0}} 2$ : Nivel de satisfacción de los pacientes en el proceso de admisión que brindó el personal de enfermería en el Servicio de Traumatología del Hospital Interzonal San Juan Bautista

El 33,70\% de pacientes valoraron que fue buena la respuesta de los auxiliares frente a su solicitud de calmar el dolor, y en menor porcentaje refieren la respuesta dada por los 
enfermeros y los licenciados ( $12,83 \%$ y $9,13 \%$ respectivamente).

En la consulta sobre las explicaciones que brinda enfermería de los procedimientos los pacientes valoraron como bueno en la mayoría de los casos del personal analizado $(59,73 \%$ de los auxiliares, el 66,39\% de los enfermeros y $60 \%$ de los licenciados).

En la figura 3 se presenta una síntesis de los puntajes obtenidos en las 460 observaciones de los procedimientos evaluados a partir de la guía de observación de las acciones que dedica el personal de enfermería a los pacientes con lesiones externas.

Se destaca que, en aquellos procedimientos del personal de enfermería que son reservados en la práctica profesional en cuanto son inherentes y específicos de su práctica, tales como la higiene del paciente y su unidad de internación, y las eliminaciones de aparato urinario y digestivo del paciente, predominaron los puntajes negativos, respecto a los otros procedimientos evaluados. Esta tendencia está representada por las acciones de los auxiliares y los enfermeros, no así en los licenciados donde los puntajes dieron positivos $(\mathrm{p}<0,05)$.

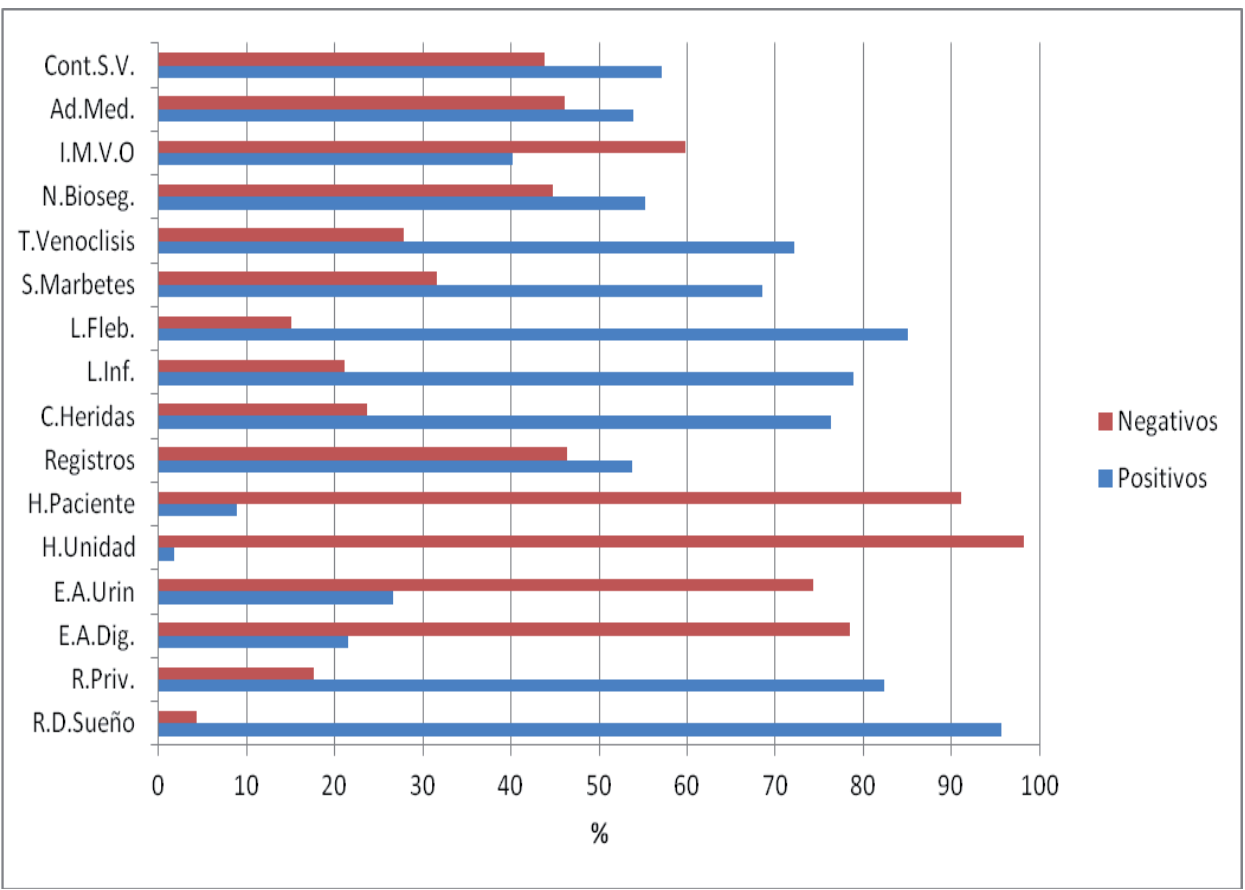

Figura $\mathrm{N}^{\mathrm{o}}$ 3: Distribución de frecuencias observadas al personal de enfermería del Servicio de Traumatología de Hospital Interzonal San Juan Bautista

\section{Discusión}

El cuidar es una actividad que ha existido desde el inicio de la humanidad, pues es algo innato y fundamental en la vida de las personas, pero no por esto se puede decir que todas las personas que cuidan a otras están haciendo enfermería. Siguiendo a Colliere "Cuidar es un acto de vida que significa una variedad infinita de actividades dirigidas a mantener la vida y permitir la continuidad y la reproducción".

Desde el punto de vista sociodemográfico el grupo etáreo que tuvo un mayor porcentaje, fue el grupo de 33 a 42 años $(34,8 \%)$, lo cual también ha sido obtenido por diversas investigaciones realizadas en América Latina que manifiestan este rango de edad como el más predominante dentro del personal de enfermería en los sistemas públicos, siendo la media de acuerdo al CIE (Consejo Internacional de Enfermeras) 42 años, el envejecimiento 
de la fuerza laboral de enfermería es también un factor a tener en cuenta en los servicios de traumatología donde la mayoría de los pacientes necesitan de la colaboración de enfermería para satisfacer sus necesidades básicas (20).

Los signos vitales son variables fisiológicas de la salud del paciente, es así, que la presión arterial y el pulso, indican el estado del sistema cardiovascular, las frecuencias respiratorias, reflejan el funcionamiento del aparato respiratorio y la temperatura corporal, el personal de enfermería en cada turno debe realizar el control de todos los signos vitales, para valorar el estado de salud de cada paciente, pero a pesar de la importancia de los mismos, un elevado porcentaje (44\%) del personal en estudio no controlan en forma completa las constantes vitales, mas aun que es una actividad independiente de enfermería y forma parte de la valoración integral del paciente, siendo una actividad prioritaria y de gran valor para la seguridad del paciente, en el trabajo la evaluación de la Calidad de los signos vitales como indicador del proceso en la Gestión del cuidado de Enfermería, muestra que el 63,67\% del personal de enfermería del instituto médico de la ciudad de México, muestra que los enfermeros no cumplen con los criterios mínimos indispensable de las normas de control de signos vitales, así mismo, hay estudios que muestran que las fallas relacionadas con eventos adversos pueden ser ocasionadas por errores en la medición y registro de las constantes vitales.

Otro aspecto que se valoró fue la administración de medicamentos, la que comprende unas series de fases hasta que llega el medicamento al paciente, en este estudio el 53,91\% del personal lo realizaron en forma correcta, a pesar que es un objetivo estratégico y un valor principal de la atención al paciente en todos los niveles del sistema de salud, los medicamentos contribuyen de manera considerable a mejorar la calidad de vida de las personas con problemas de salud, sin embargo, su uso no está exento de riesgos (21). Si no se cumple con una administración segura, puede ponerse en riesgo la recuperación de los pacientes internados y repercute en la calidad de atención que se les brinda.

Las legislaciones que regulan el ejercicio de la profesión, contemplan en las reglamentaciones que el personal de enfermería es el responsable de administrar el medicamento, es el último eslabón del sistema de medicación, por lo cual debe tener en cuenta la responsabilidad de la tarea que tiene ante sí, no solo como una tarea técnico profesional, además como un acto de conciencia social extrema y humana (22).

Algunos estudios realizados durante los últimos años muestran la presencia de errores de medicación. Las causas pueden estar relacionadas con los factores individuales del personal, como la falta de atención, deficiencia en la formación, inexperiencia, pero también con fallas sistémicas, como la iluminación, falta de personal, dificultades de comunicación (23).

En este estudio se observa que sólo el 53,91\% del personal observado aplican las normas de administración de medicamentos en forma completa, desde la planificación de las actividades, organización de la medicación, verificación de las prescripciones, preparación de los medicamentos, como ser la disolución de las presentaciones en solutos, cálculos de velocidad de goteo para los medicamentos que se administran por vía intravenosa y en forma paralela con el plan de hidratación parenteral. Asimismo cuando se analizó la aplicación correctas de normas de bioseguridad, también se detectó que solo el 53,91\%, aplicó las precauciones universales para prevenir las Infecciones intrahospitalarias, entre las cuales la principal es la higiene de manos, ya que es el proceso que se lleva a cabo para eliminar el mayor numero de microorganismos de las manos por medio de la limpieza mecánica con productos antisépticos antes y después de brindar cuidados a un paciente, o de realizar algún procedimiento. Este procedimiento constituye el método más importante, sencillo y efectivo para reducir la transmisión de microorganismos entre pacientes, la diseminación de las infecciones institucionales por contacto directo a través de las manos es la forma más común de la transmisión, por lo tanto el lavado de manos es un procedimiento esencial y debe tomarse como un indicador de calidad mensurable para conocer la eficiencia con que se realiza al brindar atención a los pacientes. Por otra parte, 
el lavado de manos ha demostrado ser efectivo en la reducción de la morbi - mortalidad por las infecciones hospitalarias, así como de los costos generados por internaciones prolongadas (24).

En relación a este tema es pertinente tener en cuenta que la Organización Mundial de la Salud publicó una guía para la higiene de manos, durante la atención institucional de pacientes denominada "Mis cinco momentos para la higiene de manos" (25), en la cual se promueve la fricción antiséptica con alcohol como el eje de las técnicas de higiene: antes del contacto con el paciente, antes de realizar una tarea aséptica, después del riesgo de exposición a líquidos corporales, después del contacto con el paciente, después de contacto con el entorno del paciente. Precisamente el reporte hace mención que al aplicar la estrategia en 55 departamentos de 43 hospitales en seis lugares de Costa Rica, Italia, Malí, Pakistán y Arabia Saudita, durante dos años desde Diciembre de 2006 a Diciembre de 2008, se observaron mejorías en las prácticas, con un incremento del $51 \%$ al $67 \%$ al finalizar la aplicación de la guía. El estudio puso de manifiesto además, que esos cambios observados en las prácticas y la cultura de seguridad se mantuvieron durante dos años al menos tras concluir la fase de prueba (25).

Al analizar la administración de soluciones intravenosas se observó que el 71,96\% del personal realizó un correcto control. Esto acuerda con la estrategia estipulada por Davini (2003) lo cual incluye un conjunto de actividades, para disponer de una solución para fluidoterapia endovenosa contenidas en envases estéril, y a la cual se le pueden adicionar medicamentos bajo condiciones asépticas, en la búsqueda de analizar la calidad del cuidado enfermero a través de indicadores que contempla la identificación de riesgos, el monitoreo de reacciones adversas, es así que la terapéutica intravenosa es un proceso de alta frecuencia e impacto en el tratamiento médico, el proceso de la preparación de la solución, e instalación es responsabilidad de enfermería (26).

La relevancia de la preparación correcta de las soluciones intravenosa, es evidente, ya que las complicaciones, puede ir desde la falla del logro de los objetivos del tratamiento, inflamaciones, infecciones tales como flebitis, bacteriemia, endocarditis, la más grave es la sepsis. (27).

El acceso vascular conlleva riesgos potenciales relativos a complicaciones iatrogénicas genéricas, pero, por sobre todo riesgos relativos a infecciones del torrente sanguíneo. Aproximadamente, el $60 \%$ de las infecciones del torrente sanguíneo se producen como consecuencia de algún tipo error en la venopunción. Así, diversos estudios realizados hace más de 10 años, indican que las infecciones del torrentes sanguíneo son responsables de una tasa extra de mortalidad que asciende al $35 \%$, asociadas a la venopunción en pacientes, tiene estrecha relación con un incremento en la duración de la internación, así como en los costos adicionales asociados a la salud con un elevado costo (28)

El proceso de admisión desde el punto de vista de atención de enfermería, consiste en la preparación de la unidad del paciente, ubicar al paciente en su unidad de acuerdo al género, estado general y patología presentarse y presentarle el compañero de sala. Valorar el estado del paciente, de los signos vitales, priorizar las necesidades que presenta. Teniendo en cuenta que la hospitalización es siempre causa de problemática psicosocial ya que el paciente pasa a una condición de pasividad, alejado de sus hábitos y costumbres enfrentando a un futuro incierto donde caben el dolor y la muerte, por eso necesita que en la recepción se le brinde un ambiente cálido y amable contribuye a brindar seguridad atenuando el temor, la ansiedad que produce la internación, en apoyo que le proporcionará enfermería, además de una atención física eficiente y segura, oportunidades para que pueda expresar sus sentimientos libremente y atenuar así sus miedos y ansiedades (29). Al respecto, es de destacar que considerar la totalidad de la persona, aun cuando el problema físico sea prioritario e inminente, debe ser objetivo de los cuidados de enfermería y la forma como reciba e introyecte ese cuidado daría respuesta a la percepción que tenga el paciente con respecto a los comportamientos del cuidado que recibe (30). En función de los expuesto y en relación a la presente investigación, se observó que el 58,91\% de los 
pacientes percibieron como bueno la recepción que ofreció el personal enfermería.

La satisfacción de los pacientes se constituye como uno de los componentes más importantes en el momento de medir la calidad de los servicios sanitarios, tal y como afirma Donabedian ; "resulta imposible describir correctamente la calidad sin contar con el punto de vista del paciente", ya que su opinión nos proporciona información acerca del éxito o fracaso del sistema sanitario para cubrir sus expectativas.

Finalmente se puede concluir que la evaluación de la calidad de los cuidados de enfermería y la satisfacción de los pacientes permitió la identificación de potencialidades y deficiencias en las actividades asistenciales. Los resultados obtenidos se constituyeron en un insumo de valor para generar estrategias que fortalezcan la calidad de los cuidados de enfermería de la institución hospitalaria.

\section{Bibliografía}

1. Gerson, R. Cómo Medir la Satisfacción del Paciente. Editorial Iberoamérica S.A. de C.V. Editor Grepe, Nicolás impreso en México. 2004 pág. 12-41.

2. Coimbra, R. Hoyt, D. La Enfermedad Trauma. Sociedad Argentina de Medicina y Cirugía del Trauma -Trauma Prioridades. Editorial Médica, Panamericana Buenos Aires. 2002 XV 23.

3. Hoyt. D., Hollingsworth, F. Quality improvements in Trauma Systems. 4 ed. Mosby. Inc. St. Louis 1999 pág. 23-24

4. González Echeverri G. Laverde de Botero, C. Ferero Pulido,C. Agudelo García, B. Sepúlveda Díaz, H. La consolidación de una Línea de Investigación sobre Trauma. Udea.edu.co/revista spt. 00/liena.htm 2005. Pág. 5.

5. Castillo Salgado, C. Mujica, O. Loyola Elizondo, E. Soler Canela, J. Control de Enfermedades en la Población, VI Modulo de Principios de Epidemiología para el Control de Enfermedades, Segunda Edición. Organización Panamericana de la Salud. Organización Mundial de la Salud. 2002 Pág. 10.

6. Castillo Salgado, C. Mujica, O. Loyola Elizondo, E. Soler Canela, J. 2002 Control de Enfermedades en la Población, VI Modulo de Principios de Epidemiología para el Control de Enfermedades, Segunda Edición. Organización Panamericana de la Salud. Organización Mundial de la Salud. 2002 Pág. 8

7. Neira. J. Epidemiología del Trauma Sociedad Argentina de Medicina y Cirugía del Trauma -Trauma Prioridades. Editorial Médica, Panamericana - Buenos Aires. 2002 Pág. 3 -7.

8. Delgado Valencia, A., Álvarez Rodríguez, Y. Aliaga Guerra, R Calidad De Atención de Enfermería desde la Percepción del Usuario que Acude al Servicio de Emergencia del Hospital Nacional Guillermo Almerada Correa Rev. de Ciencias de la Salud. 2007.

9. Marriner Tomey, Ann. RaileAlligood, M. Modelos y Teorías en Enfermería. Quinta Edición, Madrid. Edi. An Elsevier Science Imprinet. 2003 Pág. 98 - 99.

10. Trostchansky, J. Los Sistemas de Trauma: Un Ddesafío Asistencial. editorial.pagina. WEB.Uruguay.http//WWW.s.mu.org.uy.elsmu/institución/comisiones/preacetra/ sistemas/\%20traumapdf [ 2004febrero]

11. Seigel H. Dunham C.M. Trauma the Disease of the 20 th. Century, In Seigel SH. Washington. 2002 pág. 22 -24.

12. Morugij Alejandra "Hacia la Calidad Total en el Sector Sanitario" en Temas de Enfermería Actualizado (TEA) Año 4 No 10 noviembre - Diciembre 2004.

13. Neira. J. Control de Calidad en Trauma. Sociedad Argentina de Medicina y Cirugía del Trauma -Trauma Prioridades. Editorial Médica, Panamericana - Buenos Aires. 2002 pág. $60-63$

14. Cebe, L. Estrategia para mejorar la productividad en Instituciones de Salud: Cuadro 
de mando integral como herramienta de gestión. Revista ISALUD Volumen 2. 2007 Pág. $31-38$.

15. Servicio Andaluz de Salud - Manual Sistema de control de Gestión Hospitalaria, Junta de Andalucía - consejería de Salud. Enero 2005.

16. Kaplan, R. y Norton D. "Using The Balanced Scorecard As. A. Strategic Management Sistems. Harvard Business Review Boston. 2006

17. Levitt, M.S. Problems of Efficiency In: Hansen M.M. ed. The Economics of medical care. Ed. George Allen and unaurm Ltd. EE.UU. 2002.

18. Cometo, M.; Gómez, P.; Morcon Dal Sasso, G.; Enfermería y Seguridad de los Pacientes. OPS. ISBN 978-927533246-7. Año 2011

19. Ministerio de Salud y Acción Social de la Nación. La garantía de calidad en el hospital público de autogestión: Marco conceptual, estrategias e instrumentos operativos. OMS. Editorial Médica Panamericana.2006

20. Keith, F. et all. Maceration of the skin and wound bed 1: Its nature and causes JOURNAL OF Wound care 2002 Vol.11 N 7 .

21. Álvarez, C., Sossa, MP. López, L., Ortiz Ruiz, G. 2005 Impacto del uso del alcohol glicerinado en el comportamiento de la infección hospitalaria en una unidad médico - quirúrgico de cuidados intensivos. 2005 pág. $70-78$.

22. Dedrick R, Sinkowitz, Cocharan R. Cunninghan C., Mudern, R. 2007 Et al Hand Hygiene practices after briet encounters With patients an important opportunity for prevention, infec control hosp Epidemiol 2007 pág. 341 -346

23. Malagón Londoño; G., Álvarez Moreno, C.; Infecciones Hospitalarias, Editorial Panamericana, $3^{\text {a }}$. Edición Bogotá Colombia. 2010

24. Salazar Maya A., Martínez de Acosta, C., Un Sobrevuelo por Algunas Teorías donde la Interacción Enfermera - Paciente es el núcleo del Cuidado. Av. Enfermer. 2008 pág.25.

25. Organización Panamericana de la Salud (OPS/OMS). Enfoque por la Seguridad del Paciente, Washington. 2007

26. Davini, MC Guía de Evaluación Diagnóstica, Programa de Educación en Servicio, Componente de Capacitación; PROMIN Argentina 2003.

27. Luzuriaga, T., Casco M., de Capacitación y Evaluación: Transformando las Prácticas. Corriente: PROMIN. 2003.

28. Ortega Vargas, M., Leija Hernández, C. Manual de evaluación de la calidad del servicio de enfermería, Editorial Panamericana 3era. Edición. 2014

29. Moreno, V., Guevara, B., Cuidado de Enfermería al Paciente Politraumatizado un enfoque desde el Modelo de Virginia Henderson. Revista Médica Electrónica Portales Medicos.com. 2016.

30. Barragán Becerra, J., Moreno, M., Calidad percibida por usuarios de enfermería en tres hospitales públicos. Enfermería Global. Vol. 12. No 29. Murcia ene. 2013.

31. Troncoso Poblete; M., Valenzuela Suazo, S., Cuidado Humanizado: un desafío para las enfermeras en los Servicios hospitalarios. Editorial Talca. Chile 2010. 